\title{
Angiogenesis-related ultrastructural changes to capillaries in human skeletal muscle in response to endurance exercise
}

\author{
Oliver Baum, ${ }^{1}$ Jennifer Gübeli, ${ }^{1}$ Sebastian Frese, ${ }^{2}$ Eleonora Torchetti, ${ }^{1}$ Corinna Malik, ${ }^{1}$ Adolfo Odriozola, ${ }^{1}$ \\ Franziska Graber, ${ }^{1}$ Hans Hoppeler, ${ }^{1}$ and Stefan A. Tschanz ${ }^{1}$ \\ ${ }^{1}$ Institute of Anatomy, University of Bern, Bern, Switzerland; and ${ }^{2}$ Department of Neurology, University Hospital Zurich, \\ Zurich, Switzerland
}

Submitted 15 July 2015; accepted in final form 15 September 2015

\begin{abstract}
Baum O, Gübeli J, Frese S, Torchetti E, Malik C, Odriozola A, Graber F, Hoppeler H, Tschanz SA. Angiogenesis-related ultrastructural changes to capillaries in human skeletal muscle in response to endurance exercise. J Appl Physiol 119: 1118-1126, 2015. First published September 17, 2015; doi:10.1152/japplphysiol.00594.2015.The ultrastructure of capillaries in skeletal muscle was morphometrically assessed in vastus lateralis muscle (VL) biopsies taken before and after exercise from 22 participants of two training studies. In study 1 (8 wk of ergometer training), light microscopy revealed capillaryfiber $(\mathrm{C} / \mathrm{F})$ ratio $(+27 \%)$ and capillary density $(+16 \%)$ to be higher $(P$ $\leq 0.05)$ in postexercise biopsies than in preexercise biopsies from all 10 participants. In study 2 (6 mo of moderate running), $\mathrm{C} / \mathrm{F}$ ratio and capillary density were increased $(+23 \%$ and $+20 \%$; respectively, $P \leq$ $0.05)$ in VL biopsies from 6 angiogenesis responders (AR) after training, whereas 6 nonangiogenesis responders (NR) showed nonsignificant changes in these structural indicators $(-4 \% /-4 \%$, respectively). Forty capillary profiles per participant were evaluated by point and intersection counting on cross sections after transmission electron microscopy. In study 1, volume density $(\mathrm{Vv})$ and mean arithmetic thickness (T) of endothelial cells (ECs; $+19 \% /+17 \%$, respectively) and pericytes (PCs; $+20 \% /+21 \%$, respectively) were higher $(P \leq$ $0.05)$, whereas $\mathrm{Vv}$ and $\mathrm{T}$ of the pericapillary basement membrane (BM) were $-23 \% /-22 \%$ lower $(P \leq 0.05)$, respectively, in posttraining biopsies. In study 2, exercise-related differences between AR and NR-groups were found for $\mathrm{Vv}$ and $\mathrm{T}$ of PCs $(\mathrm{AR},+26 \% /+22 \%$, respectively, both $P \leq 0.05$; NR, $+1 \% /-3 \%$, respectively, both $P>$ $0.05)$ and $\mathrm{BM}(\mathrm{AR},-14 \% /-13 \%$, respectively, both $P \leq 0.05$; NR, $-9 \% /-11 \%$, respectively, $P=0.07 / 0.10)$. $\mathrm{Vv}$ and $\mathrm{T}$ of ECs were higher $(\mathrm{AR},+16 \% /+18 \%$, respectively; NR, $+6 \% /+6 \%$, respectively; all $P \leq 0.05$ ) in both groups. The PC coverage was higher $(+13 \%, P \leq 0.05)$ in VL biopsies of individuals in the AR group but nonsignificantly altered $(+3 \%, P>0.05)$ in those of the NR group after training. Our study suggests that intensified PC mobilization and $\mathrm{BM}$ thinning are related to exercise-induced angiogenesis in human skeletal muscle, whereas training per se induces EC-thickening.
\end{abstract}

morphometry; electron microscopy; capillary; skeletal muscle biopsies

IT IS WELL ESTABLISHED THAT skeletal muscles have the potential to adapt in structure and function to endurance exercise. In humans, most studies addressing this plasticity of skeletal muscle have focused on the adaptive capacity of the fibers per se, which increase their volume density $(\mathrm{Vv})$ of mitochondria (20) and store more lipid droplets (29) and glycogen (23) after a period of endurance exercise. Likewise, the microvascular system in skeletal muscle of humans may respond to a training stimulus, in particular by increasing the numerical density of the capillaries $(1,19,22)$. This process is referred to as

Address for reprint requests and other correspondence: Oliver Baum, Institute of Anatomy, Univ. of Bern, Baltzerstrasse 2, CH-3009 Bern, Switzerland. angiogenesis, which is a frequently occurring phenomenon in differentiated skeletal muscle to enhance performance (21).

Deduced from their ultrastructural features, two modes of capillary growth have been identified in skeletal muscle of rats and mice: sprouting and splitting angiogenesis [the latter mode appears to be similar or identical to intussusception (10)]. Both angiogenic modes differ significantly in their initiation and implementation.

Sprouting is triggered by the paracrine activation of capillary endothelial cells (ECs) (36), which induces their proliferation and the expression of higher levels of matrix metalloproteinases (MMPs). These MMPs catalyze the degradation/loosening of the surrounding basement membrane (BM), thereby permitting the abluminal outgrowth of new capillary segments (27). The process of outgrowth is arrested when the sprouts make contact and merge with existing capillaries (11).

Splitting/intussusceptive angiogenesis, on the other hand, is achieved by the intraluminal splitting of capillaries $(15,35)$. It is triggered by the increase in capillary shear stress that follows upstream arterial vasodilation (9). In response to the hemodynamic stimulus, ECs send cytoplasmic projections into the capillary lumen (35). When two such projections extending from opposite sides of the lumen meet, a transluminal pillar is formed, which increases in girth by the deposition of connective tissue until it separates the vascular segment into two independent microvessels. In contrast to abluminal sprouting, intraluminal splitting does not immediately depend on proliferation of capillary ECs (17).

Several experimental setups have been developed to induce angiogenesis in skeletal muscles of laboratory animals $(15,21)$. Muscle overload [e.g., induced by the surgical extirpationinduced overload of a muscle agonist (17) or in response to chronic electrical stimulation (36)] elevates the contractility of the muscle fibers, which triggers the paracrine-mediated modulation of sprouting angiogenesis. Peripheral vasodilation (e.g., initiated by the administration of the $\alpha_{1}$-adrenergic receptor antagonist prazosin) leads first to functional hyperemia and then to an increase in shear stress in the capillaries (9). The strength of the force thereby generated is somehow registered by ECs of the capillaries in the skeletal muscles and controls the degree of splitting/intussusceptive angiogenesis $(3,35)$.

Although sprouting and splitting angiogenesis in skeletal muscle have been thoroughly documented in these experimental setups, surprisingly, how physiological angiogenesis in response to endurance exercise is realized in this tissue has not been studied in either laboratory animals or humans.

Our group has characterized the changes in ultrastructure of skeletal muscle in humans in response to endurance exercise for many years $(19,20,28,30)$. In these investigations, 
however, an assessment of the ultrastructure of the capillaries was neglected. We thus have now subjected the ultrathin sections of vastus lateralis muscle (VL) biopsies from 22 individuals before and after a training period of strenuous cycling ( $8 \mathrm{wk})$ or moderate, long-lasting running $(6 \mathrm{mo})$, which were derived from two of these earlier studies $(28,30)$, to a second morphometric analysis with the distinct focus on the ultrastructural phenotype of capillaries.

The primary aim of this investigation was to verify the hypothesis that the ultrastructure of the capillaries in human skeletal muscle changes in response to endurance exercise. Because light microscopy analysis of muscle biopsies revealed that only one half of the individuals in study 2 underwent skeletal muscle angiogenesis after training, whereas the other half did not, we had the opportunity to investigate which of the training-induced alterations of the capillary ultrastructure are related to angiogenesis in skeletal muscle of humans. Furthermore, to determine whether splitting or sprouting is involved in angiogenesis in humans muscles, the outcome of study 2 was compared with the morphometrical findings described in papers using rodents $(15,21)$, in which the concept of splitting or sprouting angiogenesis had been established.

\section{MATERIALS AND METHODS}

\section{Study Participants and Protocols}

For this analysis, sections from biopsies of the VL muscle of humans were used, which were originally collected in two training studies conducted earlier by our group $(28,30)$. Informed consent was obtained from each individual before the study began. These investigations fulfilled all criteria and ethical guidelines for the treatment of human participants that were valid at the time of study execution.

Study 1. Ten untrained men exercised on an ergometer for $8 \mathrm{wk}$ $(5 \times$ wk for $30 \mathrm{~min})$. The training intensity as previously described in detail (28) was judged by the heart rate achieved at the end of each training session, which typically reached $90-95 \%$ of each participant's maximum. In addition, the training intensity was periodically checked by continuous recording of heart rate, oxygen uptake, and plasma lactate levels. The work loads were set to allow each subject to work at peak power output, maintainable for $30 \mathrm{~min}$, and increased periodically to keep the training intensity at the same relative level throughout the training period.

The mean age of the participants was $30.5 \pm 5.0 \mathrm{yr}$, and their mean height was $178.0 \pm 7.1 \mathrm{~cm}$. The mean body mass of $70.8 \pm 5.4 \mathrm{~kg}$ was nonsignificantly altered $(P>0.05)$ during the training period. As determined by an incremental performance test on the ergometer, the mean $\dot{\mathrm{V}}_{2}$ max increased significantly $(P \leq 0.05)$ from $51.6 \pm 3.2$ $\mathrm{ml} \cdot \mathrm{kg}^{-1} \cdot \mathrm{min}^{-1}$ to $58.4 \pm 2.1 \mathrm{ml} \cdot \mathrm{kg}^{-1} \cdot \mathrm{min}^{-1}$.

Study 2. Although 20 untrained male subjects originally participated in this study (30), sections from only 12 randomly selected participants (mean age, $36.3 \pm 6.3 \mathrm{yr}$; mean height, $181.0 \pm 7.1 \mathrm{~cm}$; mean body wt, $85.6 \pm 16.0 \mathrm{~kg}$; mean body mass index, $26.2 \pm 4.0$ $\mathrm{kg} / \mathrm{m}^{2}$ ) were prepared for morphometry.

Before training, the participants had not been engaged in any endurance activities. The moderate home-based training regimen consisted of four 30-min jogging sessions per week for 6 mo, with a heart rate corresponding to a maximal oxygen uptake $\left(\dot{\mathrm{V}}_{2}\right.$ max $)$ of $75 \%$. The heart rate of each subject who participated in this study was continuously recorded during the training with a portable heart rate monitor as previously reported (30).

$\dot{\mathrm{V}}_{2}$ max was determined via a continuous incremental exercise test on a bicycle ergometer (start at $75 \mathrm{~W}$, followed by increments of 30 $\mathrm{W}$ every $2 \mathrm{~min}$ ) to voluntary exhaustion. The mean $\dot{\mathrm{V}}_{\mathrm{O}_{2} \text { max }}$ increased from a pretraining value of $38.3 \pm 6.0 \mathrm{ml} \cdot \mathrm{kg}^{-1} \cdot \mathrm{min}^{-1}$ to a posttrain- ing value of $40.3 \pm 6.3 \mathrm{ml} \cdot \mathrm{kg}^{-1} \cdot \mathrm{min}^{-1}$, but the difference was not significant $(P>0.05)$.

\section{Biopsies}

VL biopsies, 50-100 $\mathrm{mg}$ in weight, were obtained from each participant using a Bergström fine needle before and $48 \mathrm{~h}$ after the last training session, as previously described $(28,30)$. The specimens were chemically fixed in a $6.25 \%$ ( $\mathrm{vol} / \mathrm{vol})$ glutaraldehyde solution buffered with $0.1 \mathrm{M}$ sodium cacodylate- $\mathrm{HCl}(\mathrm{pH} 7.4)$ for several days at $4{ }^{\circ} \mathrm{C}$.

\section{Light Microscopy}

After chemical fixation, VL biopsies were divided into two to three pieces, each with a volume of $\sim 0.5 \mathrm{~mm}^{3}$, after which they were postfixed in $1 \%$ (wt/vol) $\mathrm{OsO}_{4}$, stained en bloc in $0.5 \%$ uranyl acetate, dehydrated in increasing concentrations (70-100\%) of ethanol, and embedded in Epon 812 (Fluka, Buchs, Switzerland).

Semithin sections (1 $\mu \mathrm{m}$ thickness) were cut on an ultramicrotome (LKB Ultrotom III, Bromma, Sweden) using a diamond knife (Diatome, Biel, Switzerland) and stained with Toluidine blue (4). For morphometric analysis, transverse or slightly oblique sections through the muscle (volume $\sim 1 \mathrm{~mm}^{3}$ ) were cut from two randomly selected Epon blocks. A systematic sampling strategy was implemented to acquire 10 photographs of each section at a magnification of $\times 630$ using a Leica DMR light microscope (Leica Microsystems, Heerbrugg, Switzerland). Using these light micrographs, the number of capillary profiles and that of muscle fibers were quantified taking into account the forbidden line rule (33). The mean cross-sectional fiber area (MCSFA) was estimated by point counting, and capillary profile density and capillary-to-fiber $(\mathrm{C} / \mathrm{F})$ ratio were calculated as previously described (4).

\section{Transmission Electron Microscopy}

Ultrathin sections (70-80 nm in thickness) of the Epon blocks were prepared with an ultramicrotome (LKB Ultrotom III), using a diamond knife (Diatome). The sections were floated on 200-mesh parlodion-coated copper grids (Plano, Wetzlar, Germany) and contrasted with $0.5 \%$ uranyl acetate and $3 \%$ lead citrate as previously described $(4,19)$. The inspection was carried out using a transmission electron microscope (Morgagni M268; FEI, Brno, Czech Republic).

\section{Morphometric Analysis}

Ultrathin sections from two randomly selected Epon-embedded blocks of each VL biopsy were used for morphometric analysis.

Twenty randomly selected capillaries from each section were photographed using the transmission electron microscope at a final magnification of $\times 8,900$ (corresponding to a $11.1 \mu \mathrm{m} \times 7.9 \mu \mathrm{m}$ field of view) using a MORADA digital camera (OSIS, Münster, Germany). Capillaries with a length-to-width ratio larger than 2 were considered to be too obliquely sectioned and were excluded from the morphometric analysis because the boundary length of the capillaries was used as a reference structure in the morphometric analysis (see below). The electron micrographs were overlaid with a grid consisting of $10 \times 8$ perpendicular test lines at a distance (d) of $1.2 \mu \mathrm{m}$ in the final magnification. The line crossings were defined as the test points (totally 80), each representing an area of $1.44 \mu \mathrm{m}^{2}(=1.2 * 1.2)$.

The number of points $(\mathrm{P})$ landing on capillary lumen, ECs, EC nucleus, pericytes (PCs), and BM were counted and expressed relative to the number of all reference points on the capillaries, thereby yielding unbiased estimates of the mean $\mathrm{Vv}\left(\mathrm{Vv}=\mathrm{P}_{\text {compartment }} /\right.$ $\mathrm{P}_{\text {capillary }}$ ) and the mean cross-sectional area (A) of each compartment as $\mathrm{A}=\mathrm{P}_{\text {compartment }} * \mathrm{~d}^{2}$.

The counting of line intersections (I) that overlapped with the abluminal side of the capillary ECs ( = EC/BM transition) permitted an estimate of the mean boundary length (B) of this border. It was calculated as $\mathrm{B}=0.5 * \mathrm{~d} / 2 * \pi * \mathrm{I}(33)$. The mean arithmetic 
thickness (T) of each compartment was calculated as the crosssectional area (representing the profile of its volume) divided by the boundary length (representing its surface density) computed as $\mathrm{T}=$ $\mathrm{P}_{\text {compartment }} * \mathrm{~d} /(0.25 * \pi * \mathrm{I})$.

The nucleus volume density in ECs was calculated as $\mathrm{P}_{\mathrm{EC}}$ nucleus $\left(\mathrm{P}_{\mathrm{EC} \text { nucleus }}+\mathrm{P}_{\mathrm{EC}}\right)$. The $\mathrm{PC}$ coverage of the capillaries was expressed as the number of test lines simultaneously crossing both the EC/BM transition and a PC surface relative to the total number of test lines hitting the EC/BM transitions without PC contact. The intraluminal filopodia proportion was calculated as the number of test lines crossing a projection surface relative to the total number of test lines hitting lumen EC/transitions.

Due to their localization within the BM around capillaries, which is easily recognized as a sharply demarcated layer on electron micrographs, we are sure to have included only PCs in the morphometry of PC features. Other cell types present in the skeletal muscle endomysium (mast cells, macrophages, lymphocytes, satellite cells, fibroblasts, telocytes, and stem cells) are not found inside the pericapillary BM. Smooth muscle cells are located in the perivascular BM but exclusively surround arterioles, arteries, and veins in skeletal muscle as a characteristic closed cell ring accompanied by an internal and external elastic lamina.

Bear in mind that all morphometric indicators are two-dimensional (2-D) approximations and do not represent unbiased estimators in $3-\mathrm{D}$.

\section{Statistical Analysis}

Numerical data are expressed as means and standard deviations. Parameters pertaining to the morphometric analyses of study 1 were compared using the Student's $t$-test.

In study 2, the significance of differences between the AR and NR groups and between pretraining and posttraining groups were assessed by a one-way ANOVA (repeated measures). In cases of significant interactions, differences in the morphometric indicators between the groups were identified using the pairwise Bonferroni correction method. Statistical significance was set at $P \leq 0.05$.

\section{RESULTS}

To study the capillarity in skeletal muscles of humans after endurance exercise, we studied the capillary supply by means of light microscopy and the ultrastructure of the capillaries by transmission electron microscopy (TEM) in combination with morphometry.

\section{Capillary Supply}

Light microscopy was performed to quantify the capillary supply in the 44 VL biopsies of 22 participants of the two training studies collected before and after endurance exercise training (Table 1). In both studies, the mean values for the $\mathrm{C} / \mathrm{F}$ ratio and capillary density were higher in the biopsies obtained after the exercise training period than in those obtained before the period. These differences in capillary supply were significantly higher $(P \leq 0.05)$ in all 10 participants in study $1(\mathrm{C} / \mathrm{F}$ ratio, $+27 \%$; capillary density, $+16 \%)$, but varied only nonsignificantly $(P>0.05)$ in the 12 participants of study $2(\mathrm{C} / \mathrm{F}$ ratio, $+9 \%$; capillary density, $+6 \%)$. By contrast, MCSFA was not significantly different $(P>0.05)$ between the VL biopsies obtained before or after exercise in either study (study 1, +8\%; study $2,+3 \%)$.

An increased $\mathrm{C} / \mathrm{F}$ ratio in skeletal muscle is characteristic for individuals considered to be AR, whereas individuals considered to be NR comprise those subjects who do not exhibit a higher $\mathrm{C} / \mathrm{F}$ ratio in response to an angiogenic stimulus. In accordance to this definition, closer analysis of the VL biopsies revealed that all participants in study 1 were ARs. In study 2, the $\mathrm{C} / \mathrm{F}$ ratio was increased after training $(23 \% ; P \leq 0.05)$ in VL biopsies of 6 AR individuals, whereas $6 \mathrm{NR}$ individuals exhibited nonsignificant changes $(-4 \% ; P \geq 0.05)$ in this indicator (Table 1).

There were no significant differences $(P \geq 0.05)$ in anthropometric data between the participants in either the AR or NR groups in study 2: age (AR, $36.2 \pm 5.3 \mathrm{yr}$; NR, $37.8 \pm 8.3 \mathrm{yr}$ ), $\mathrm{VO}_{2}$ max before training $\left(\mathrm{AR}, 39.2 \pm 6.6 \mathrm{ml} \cdot \mathrm{min}^{-1} \cdot \mathrm{kg}^{-1}\right.$; NR, $\left.39.8 \pm 5.6 \mathrm{ml} \cdot \mathrm{min}^{-1} \cdot \mathrm{kg}^{-1}\right), \dot{\mathrm{V}}_{2}$ max after training $(\mathrm{AR}, 41.2 \pm$ $\left.7.4 \mathrm{ml} \cdot \mathrm{min}^{-1} \cdot \mathrm{kg}^{-1} ; \mathrm{NR}, 41.5 \pm 6.5 \mathrm{ml} \cdot \mathrm{min}^{-1} \cdot \mathrm{kg}^{-1}\right)$, body mass (AR, 90.2 $\pm 22.3 \mathrm{~kg}$; NR, $80.9 \pm 6.0 \mathrm{~kg}$ ), height (AR, $182.1 \pm 6.6 \mathrm{~cm} ; \mathrm{NR}, 180.7 \pm 5.4 \mathrm{~cm}), \mathrm{BMI}(\mathrm{AR}, 26.3 \pm 6.0$ $\mathrm{kg} / \mathrm{m}^{2}$; $\mathrm{NR}, 25.7 \pm 0.7 \mathrm{~kg} / \mathrm{m}^{2}$ ), and mean training performance/week (AR, $13.0 \pm 4.8 \mathrm{~km}$ and $77 \pm 21 \mathrm{~min}$; NR, $16.8 \pm 3.2 \mathrm{~km}$ and $110 \pm 42 \mathrm{~min})$.

\section{Electron Microscopy}

For qualitative and morphometric analyses of their ultrastructure in VL biopsies, 40 capillaries (20 from each of two blocks) in each of the 44 biopsies ( 22 before-exercise and 22 after-exercise biopsies) were randomly assessed by TEM. Hence, in total, TEM sections of 1,760 capillary profiles were evaluated.

\section{Qualitative TEM Analysis}

In each case, the capillary profile manifested the same characteristic ultrastructural features: the lumen was mantled with ECs, and the abluminal surface of the ECs was skirted by a dense BM containing the profile of one or more PCs (Fig. 1, $A$ and $B$ ). In many of the capillaries of all participants, one or more protrusions projected from the ECs into the capillary lumen often in close proximity to tight junctions (Fig. 1C). Some ECs of capillaries, particularly in

Table 1. Capillary supply in vastus lateralis biopsies collected before and after endurance exercise*

\begin{tabular}{|c|c|c|c|c|c|c|}
\hline & \multicolumn{2}{|c|}{ Study-1 } & \multicolumn{4}{|c|}{ Study-2 } \\
\hline & Before & After & Before & After & Before & After \\
\hline Capillary density, $\mathrm{mm}^{-2}$ & $464 \pm 47$ & $534 \pm 60 \dagger$ & $435 \pm 95$ & $520 \pm 99 \dagger$ & $482 \pm 60$ & $460 \pm 52$ \\
\hline MCSFA, $\mu \mathrm{m}^{2}$ & $3,874 \pm 669$ & $4,120 \pm 484$ & $3,314 \pm 824$ & $3,458 \pm 605$ & $3,385 \pm 546$ & $3,428 \pm 804$ \\
\hline
\end{tabular}

*Values are means \pm SD as determined applying morphometry after light microscopy of semithin sections. $\dagger$ Different between pre- and postexercise. AR, angiogenesis responder; C/F, capillary-fiber; MCSFA, mean cross-sectional fiber area, NR, nonangiogenesis responder. 

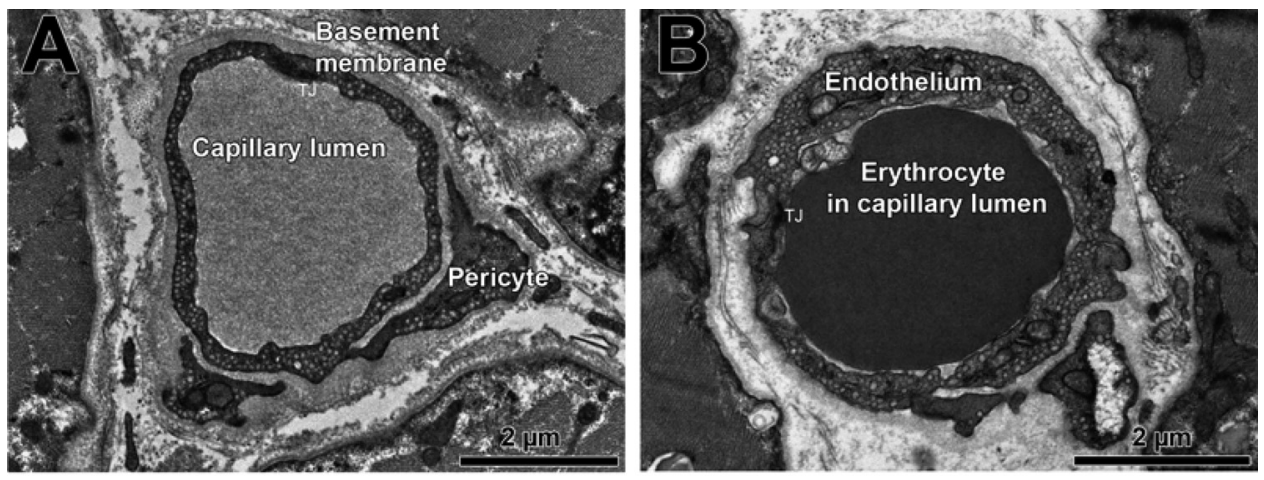

Fig. 1. Transmission electron microscopy images used to demonstrate the ultrastructure of capillaries in vastus lateralis (VL) biopsies. Shown are representative micrographs of capillaries from VL biopsies col-
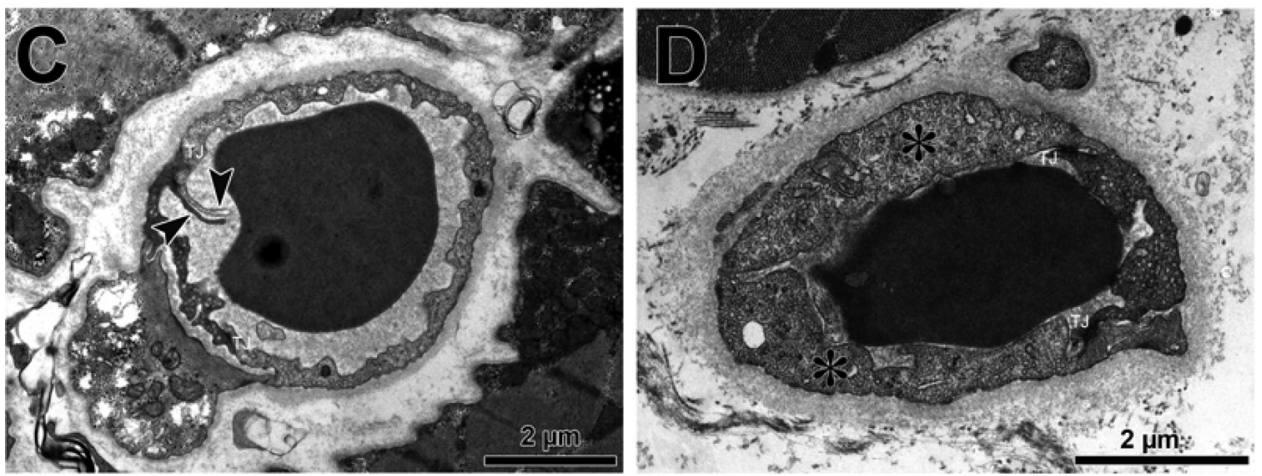
lected from a participant before $(A)$ and after $(B)$ the endurance exercise stimulus. $C$ : in most pre- and postexercise biopsies, endothelial cells (ECs) send forth projections often extending in pairs (arrowheads) into the capillary lumen. $D$ : in many instances, the ECs in capillaries of postexercise biopsies appeared enlarged (asterisks). Occasionally, we have observed capillaries with short $(E)$ or long $(F)$ abluminal projections (arrowheads) that may represent sprouts outlining the capillary lumen (asterisk). Electron micrographs were derived from participants as follows: A, study 2 NR preexercise; $B$, study 2 AR postexercise; $C$, study 1 preexercise;
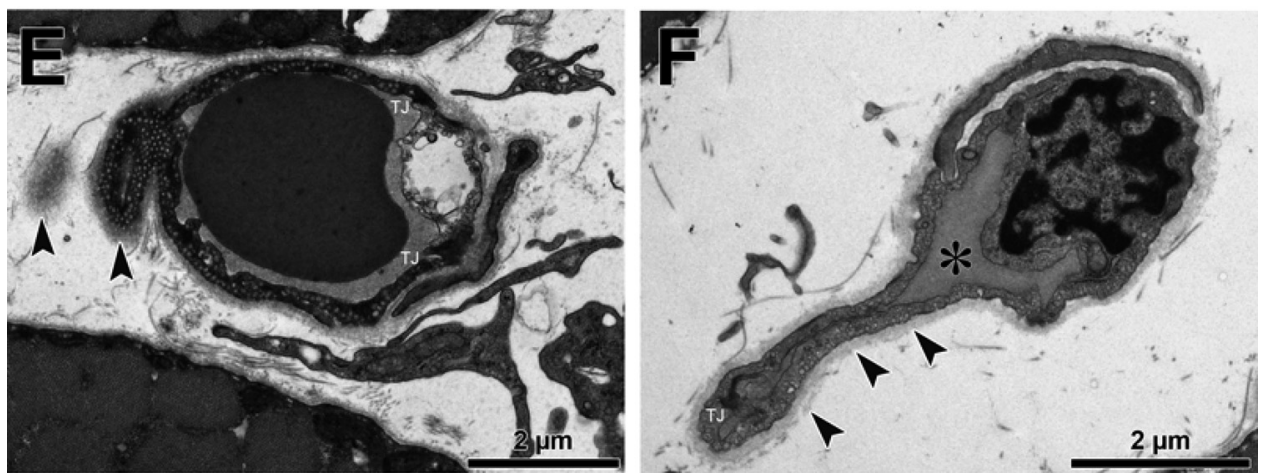
$D$, study 1 postexercise; $E$, study 2 AR postexercise; $F$, study 2 AR postexercise. TJ indicates presumptive tight junctions.

those of postexercise biopsies, were strongly enlarged and thickened (Fig. 1D).

We made use of the micrographs to study the spatial relationship between ECs and PCs in human VL biopsies. Consistently, the PC profiles were separated by a pericapillary BM from the abluminal surface of the EC (Fig. 1). Frequently, PC projections entered the ECs (Fig. 2, $A$ and $B$ ). At those sites where these PC projections invaded the ECs, either EC bridges (Fig. 2C) or intracellular EC pockets and holes (Fig. 2, $D-F$ ) were observed, as far as it is possible to assess this on 2-D photographic images. This feature of PCs invading ECs was observed in about $50 \%$ of all capillary profiles detected in VL biopsies taken before and after exercise (data not shown).

Relatively infrequently (in 2 preexercise and 6 postexercise capillary profiles out of 1,760 assessed), we detected abluminal projections growing out from the EC toward the endomysium, which we assumed to be sprouts (Fig. 1, E and $F$ ). Remarkably, the entire capillary circumference with the abluminal projections was continuously surrounded by an intact pericapillary BM. Serial sections enabled us to characterize one of these presumptive sprouts in more detail (Fig. 3): it became detached from the abluminal surface of a capillary
EC (Fig. 3C) and ended finally as a small EC clot without a detectable lumen (Fig. 3D).

Cytoplasmic vacuolization was observed to a various extent in the capillary ECs: some ECs contained only a few membranous vesicles, whereas others were completely filled by these organelles (compare Fig. 2, A vs. D). However, apparent differences in the degree of vacuolization in one of the experimental groups were not evident to us.

\section{Morphometry of the Capillary Ultrastructure}

Study 1. As shown in Fig. 4, the values for the mean arithmetic thickness $(\mathrm{T})$ of ECs $(+17 \%)$ and PCs $(+21 \%)$ were significantly higher $(P \leq 0.05)$, whereas $\mathrm{T}$ of $\mathrm{BM}$ was significantly lower $(-22 \%$, $P \leq 0.05)$ in the postexercise VL biopsies than in preexercise biopsies. T of lumen did not differ significantly $(-1 \%, P>0.05)$ between the biopsies. Accordingly, the mean cross-sectional capillary area including $\mathrm{BM}$ and $\mathrm{PCs}(+5 \%)$ and the mean arithmetic circumference $(+8 \%)$ differed nonsignificantly $(P>0.05)$ between pre- and postexercise biopsies. The relative proportion of the compartment $\mathrm{T}$ resembles the absolute estimations: the $\mathrm{Vv}$ of ECs $(+19 \%)$, PCs $(+20 \%)$, and $\mathrm{BM}(-23 \%)$ but not the lumen $(-5 \%)$ were signifi- 

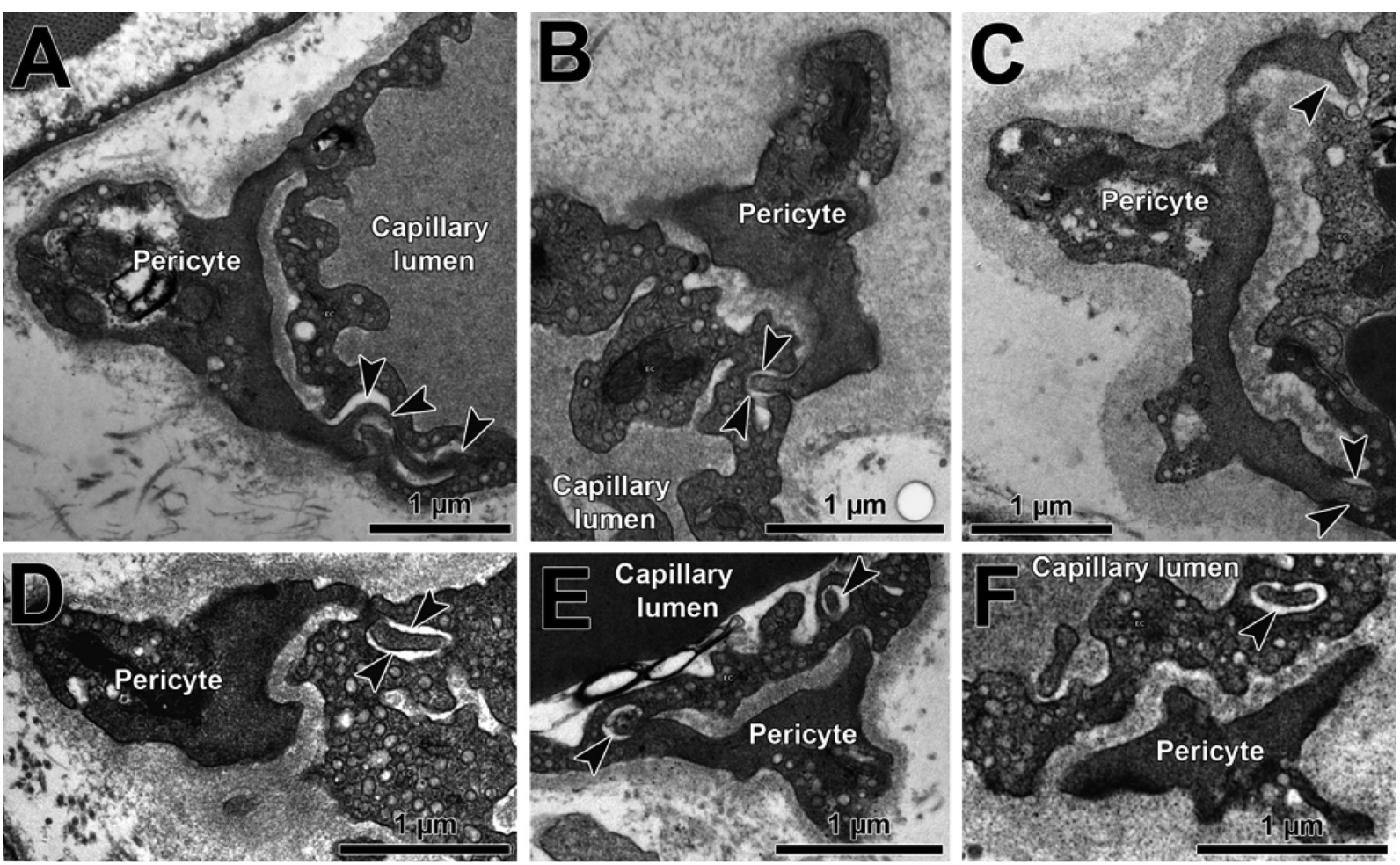

Fig. 2. Characterization of the endothelial cell (EC)-pericyte (PC) interaction. On all electron micrographs of capillaries, the abluminal side of ECs was consistently surrounded by a basement membrane in which PC profiles were frequently embedded. Projections of PCs were regularly observed entering the ECs at their abluminal surface (sockets) to a different extent $(A-F)$. Note the intracellular EC holes in $D-F$ (arrowheads), which presumably represent pockets for invading PC projections.

cantly different $(P \leq 0.05)$ between the post- and preexercise biopsies. As shown in Fig. 4, the nucleus density in ECs $(-9 \%)$ and the enlargement of the luminal EC surface by projections $(+7 \%)$ were nonsignificantly altered $(P>0.05)$ in the capillaries of the VL biopsies after the training period. In contrast, the PC coverage of the capillaries, which reflects the proportion of abluminal EC surface that is covered by PCs, was significantly increased $(+17 \%, P \leq 0.05)$ after exercise.
Fig. 3. Identification of a capillary sprout on serial ultrathin sections. Unilaterally, an endothelial cell (EC) hollow with clearly visible lumen (asterisks) grew out of the crosssectioned capillary $(A$; also see higher magnification in $B)$. On the following two serial sections $(C$ and $D)$, which were separated by $500 \mathrm{~nm}$ between each other, this abluminal EC sprout extended (arrowhead) to terminate as a small EC profile (arrow) surrounded by a basement membrane.
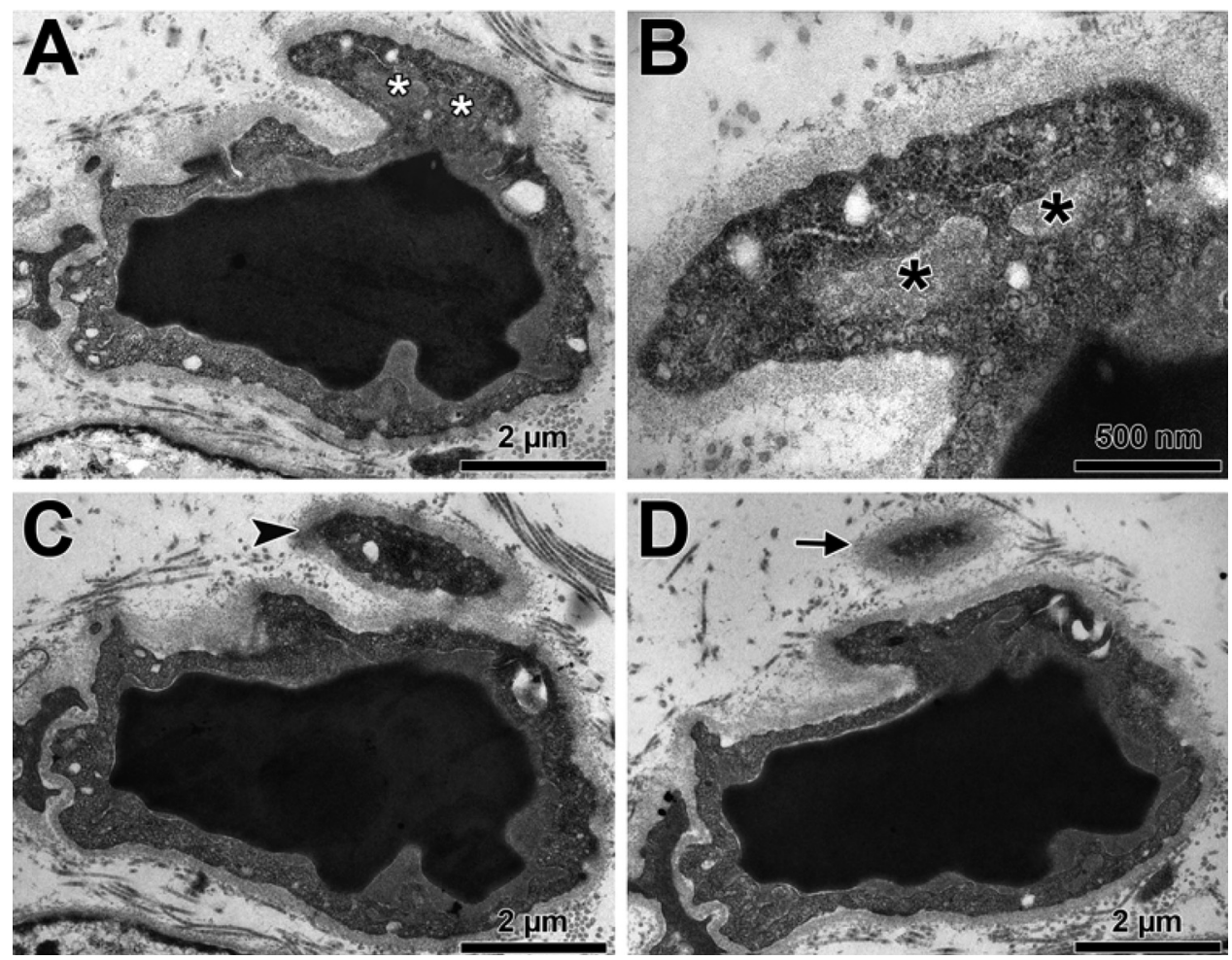

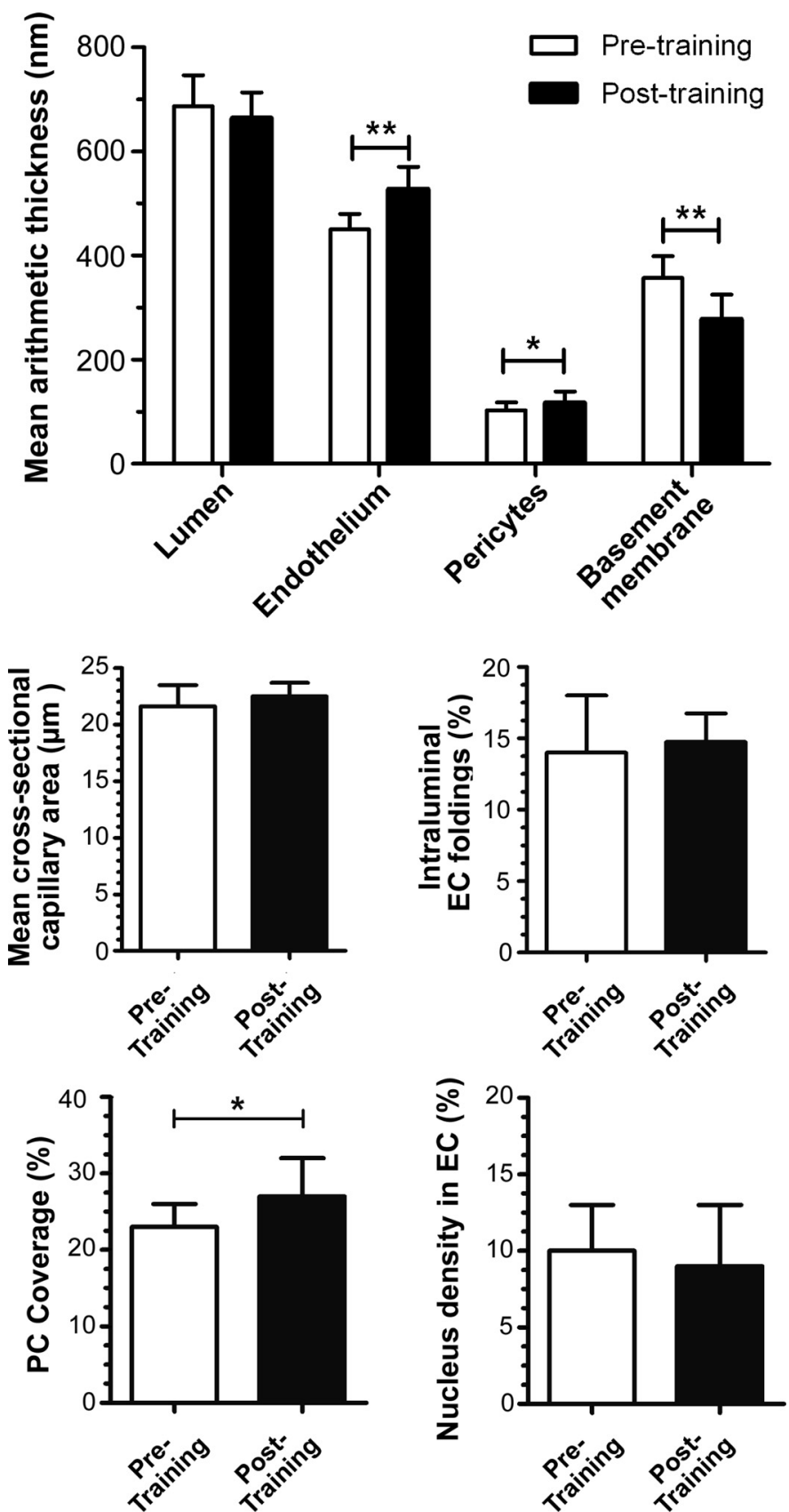

Fig. 4. Morphometry of the capillary ultrastructure from pre- and postexercise biopsies in study 1. With the outcome of the point and intersection counting performed on micrographs of capillaries from the 10 pre- and 10 postexercise biopsies from individuals in study 1 , morphometric indicators were computed. Means \pm standard deviations are represented. *Significantly different between pre- and postexercise biopsies. EC, endothelial cell; PC, pericyte.

Study 2. Subjects in study 2 underwent less strenuous training than the participants in study 1 . In the AR cohort, similar quantitative changes in the capillary ultrastructure as in study 1 were found in response to endurance exercise (Table 2).

To determine whether changes in the capillary ultrastructure in response to exercise are associated with the onset of angiogenesis, the morphometric findings of the AR and NR groups from study 2 were compared. Most indicators were altered to a similar extent in both groups by the training (Table 2).
Significant preexercise and postexercise differences between the AR and NR groups were found for $\mathrm{Vv}$ and $\mathrm{T}$ of PC (AR group, $+26 \%$ and $+22 \%$, both $P \leq 0.05$; NR group, $+1 \%$ and $-3 \%$, both $P>0.05$ ) and BM (AR group, $-14 \%$ and $-13 \%$, both $P \leq 0.05$; NR group, $-9 \%$ and $-11 \%, P=$ $0.07 / 0.10)$, whereas the EC surface enlarged by projections was significantly altered in the NR group $(+24 \% ; P \leq 0.05)$ but not the AR group $(+11 \% ; P>0.05)$. The PC coverage of the capillaries was significantly altered in the postexercise biopsies from the AR group $(+13 \% ; P \leq 0.05)$ but not the NR group $(+3 \% ; P>0.05)$.

$\mathrm{Vv}$ and T of EC were significantly higher (AR group, $+16 \%$ and $+18 \%$, both $P \leq 0.05$; NR group, $+6 \%$ and $+6 \%$, both $P \leq$ $0.05)$ in the posttraining than the pretraining VL biopsies of both groups, suggesting that EC thickening was induced by the training stimulus as such without association with angiogenesis.

\section{DISCUSSION}

In the present investigation, we morphometrically assessed the ultrastructure of capillaries in $44 \mathrm{VL}$ muscle biopsies taken before and after exercise from 22 participants in two training studies. In study 1, all 10 participants underwent angiogenesis in skeletal muscle. In study 2, 6 AR individuals were identified, whereas six NR individuals did not show a significant change in $\mathrm{C} / \mathrm{F}$ ratio after training. TEM analysis combined with morphometry of capillaries in VL biopsies of AR and NR individuals in study 2 suggest that intensified PC mobilization and pericapillary BM thinning are related to exercise-induced angiogenesis, whereas training per se induced EC thickening. Comparison of the structural indicators assessed in our analysis of human skeletal muscle biopsies with those gained in rodents, as previously published, does not provide clear indication whether exercise-induced angiogenesis in humans is implemented by sprouting, or splitting, or both.

Angiogenesis in skeletal muscle is defined as a statistically significant increase in $\mathrm{C} / \mathrm{F}$ ratio values (21). Although we included two blocks (with 10 micrographs each) per biopsy in the morphometric analysis, $\mathrm{C} / \mathrm{F}$ ratio was calculated after addition of all capillary and fiber profiles on the 20 micrographs. With only one reading of capillary supply per biopsy, however, statistical significance cannot be computed and, thus it cannot be decided whether training led to induction of angiogenesis in the skeletal muscle of a particular person. To overcome this problem, we defined $\mathrm{AR}$ as if the $\mathrm{C} / \mathrm{F}$ ratio was at least $10 \%$ higher in the postexercise biopsies than in the preexercise biopsies. All other participants were assigned to the NR group. This threshold used to allocate the participants in study 2 into AR and NR groups is mainly based on experimental experiences with elite athletes and untrained volunteers $(28,30)$. In both preexercise and postexercise biopsies of the cohorts in these studies, changes in the $\mathrm{C} / \mathrm{F}$ ratio lower than about $10 \%$ were revealed to be statistically nonsignificant, as also has been reported by others (18). This value was then transferred as a threshold to the individual analyses described here. Using this classification, we could identify 10 AR (study 1) and 6 AR and $6 \mathrm{NR}$ (study 2) individuals who contributed to our investigation.

Although all participants in study 2 underwent exercise training of similar intensity and duration, the changes in capillary supply in the VL were inconsistent. Heterogeneity in 
Table 2. Quantitative stereological analysis of capillary ultrastructure of angiogenesis responders and nonangiogenesis responders in study 2

\begin{tabular}{|c|c|c|c|c|}
\hline & \multicolumn{2}{|c|}{ AR, $n=6$} & \multicolumn{2}{|c|}{$\mathrm{NR}, n=6$} \\
\hline & Before & After & Before & After \\
\hline A (cap with BM and PC), $\mu m^{2}$ & $23.4 \pm 2.2$ & $23.6 \pm 2.6$ & $22.3 \pm 2.2$ & $21.6 \pm 2.2$ \\
\hline Vv(lumen cap), \% & $0.39 \pm 0.03$ & $0.36 \pm 0.04$ & $0.37 \pm 0.03$ & $0.37 \pm 0.02$ \\
\hline $\mathrm{Vv}(\mathrm{EC} ;$ cap $), \%$ & $0.28 \pm 0.02$ & $0.32 \pm 0.03 \dagger$ & $0.30 \pm 0.03$ & $0.32 \pm 0.03 \dagger$ \\
\hline $\mathrm{Vv}(\mathrm{PC} ;$ cap $), \%$ & $0.09 \pm 0.02$ & $0.11 \pm 0.02 \dagger$ & $0.10 \pm 0.01$ & $0.10 \pm 0.01$ \\
\hline $\mathrm{Vv}(\mathrm{BM} ;$ cap $), \%$ & $0.24 \pm 0.04$ & $0.21 \pm 0.03 \dagger$ & $0.23 \pm 0.02$ & $0.21 \pm 0.03$ \\
\hline Intraluminal projection proportion, $\%$ & $0.11 \pm 0.04$ & $0.12 \pm 0.03$ & $0.11 \pm 0.03$ & $0.13 \pm 0.02 \dagger$ \\
\hline PC coverage, $\%$ & $0.24 \pm 0.03$ & $0.27 \pm 0.03 \dagger$ & $0.26 \pm 0.03$ & $0.27 \pm 0.03$ \\
\hline EC nucleus density, $\%$ & $0.08 \pm 0.04$ & $0.12 \pm 0.03$ & $0.10 \pm 0.04$ & $0.12 \pm 0.02$ \\
\hline $\mathrm{T}$ (lumen), nm & $741 \pm 32$ & $680 \pm 63$ & $718 \pm 69$ & $710 \pm 33$ \\
\hline $\mathrm{T}(\mathrm{EC}), \mathrm{nm}$ & $422 \pm 20$ & $496 \pm 54 \dagger$ & $452 \pm 32$ & $478 \pm 28 \dagger$ \\
\hline $\mathrm{T}(\mathrm{PC}), \mathrm{nm}$ & $151 \pm 35$ & $184 \pm 28 \dagger$ & $155 \pm 22$ & $151 \pm 22$ \\
\hline $\mathrm{T}(\mathrm{BM}), \mathrm{nm}$ & $388 \pm 62$ & $339 \pm 41 \dagger$ & $355 \pm 43$ & $316 \pm 58$ \\
\hline $\mathrm{B}(\mathrm{cap}), \mu \mathrm{m}$ & $18.3 \pm 0.9$ & $18.7 \pm 1.4$ & $17.5 \pm 0.8$ & $17.2 \pm 1.1$ \\
\hline
\end{tabular}

Transmission electron micrographs of transversally sectioned 960 capillaries in 12 vastus lateralis biopsies of participants in study 2 collected before and after training sessions were subjected to a morphometric analysis of the listed structural indicators by point and intersection counting. Values are means \pm SD. A, area; B, mean arithmetic circumference; BM, basement membrane; cap, capillary; EC, endothelial cell; PC, pericyte; T, mean arithmetic thickness; Vv, volume density. $\dagger P \leq 0.05$ between pre- and postexercise biopsies.

responsiveness to a training stimulus is a well-documented phenomenon for other parameters (e.g., $\dot{\mathrm{V}}_{\mathrm{O}_{2} \max }$ ) and is assumed to reflect mainly individual differences in genetic predisposition $(7,24,25)$, which could be ascribed, for example, to the variable frequency of transcriptional reading of the VEGF promoter (26). The evident variances in angiogenic capacity might be likewise ascribed to the heterogeneous genetic background of the participants but it might be also influenced by their health status and their distinct fitness (untrained individuals in study 2), or by the training load (rather moderate in study 2 compared with that in study 1).

To the best of our knowledge, this is the first study in which the ultrastructural phenotype of capillaries has been evaluated in skeletal muscles of nondiseased, young humans after aerobic training. Notably, a partial aspect of this topic was addressed in reporting that the thickness of the BM was reduced by $30-$ $40 \%$ in the gastrocnemius muscle of elderly men (mean age 64 yr) after 9 mo of endurance exercise training (34).

In both studies, the VL muscle biopsies were taken 2 days after the last training session. Consequently, ultrastructural alterations of capillaries, which we describe here, are not caused acutely, but represent chronic changes in the capillary arrangement.

In study 1, ECs and PCs showed a relative (volume density; $\mathrm{Vv}$ ) and absolute (mean arithmetic thickness; T) increase, whereas $\mathrm{Vv}$ and $\mathrm{T}$ of the pericapillary BM were lower in postexercise biopsies than in preexercise biopsies from study 1 individuals. In contrast, $\mathrm{Vv}$ and $\mathrm{T}$ of the capillary lumen were not significantly altered in the VL biopsies after training. Because the area and the circumference of capillaries in toto did not differ significantly between the pre- and postexercise biopsies, quantitative changes in $\mathrm{Vv}$ and $\mathrm{T}$ in response to the training stimulus must be caused by an internal rearrangement of the capillary compartments (EC, BM, PC).

To determine whether any of these exercise-related changes of the capillary ultrastructure are specifically associated with angiogenesis or rather represent angiogenesis-unrelated reactions of the microvasculature to the exercise stimulus, we assessed and compared the phenotype of the capillaries in skeletal muscles of AR and NR individuals in study 2 by means of morphometry. Most strikingly, higher values for $\mathrm{Vv}$ and $\mathrm{T}$ of PC and lower ones of BM as well as increased PC coverage were detected in the biopsies of the AR group but not in those of the NR group, indicating that higher PC coverage and BM thinning are related to exercise-dependent angiogenesis in humans. In contrast, the thickening of ECs after exercise was observed in the VL biopsies of both groups. Thus EC expansion appears to be unrelated to angiogenesis. Interestingly, EC swelling has also been observed in rats subjected to electrical stimulation (12), which resembles an experimental procedure of endurance exercise.

Hudlicka, Egginton, Brown, and colleagues (13, 15, 35, 36) have identified hallmarks for changes in the ultrastructural phenotype of capillaries associated with splitting and sprouting angiogenesis in skeletal muscles of rodents. However, it is not known how this process is physiologically realized in response to endurance exercise. This lack of knowledge is at least partly due to the fact that there are no cellular markers available for either angiogenic mode so far. Without doubt, the gold standard to address this issue is therefore still the quantitative evaluation of the capillary ultrastructure by the combination of TEM analysis and morphometry $(13,15,32,35,36)$, which, however, is complicated to perform.

During splitting angiogenesis, a higher proportion of intraluminal irregularities, projections, and septa combined with extensive cytoplasmic vacuolization of ECs were observed in skeletal muscle capillaries of prazosin-treated rats compared with those of control animals (35). In contrast, we found the intraluminal EC surface in capillaries to be nonsignificantly altered by projections in the postexercise biopsies of the study participants. These findings implicate that physiological angiogenesis in skeletal muscles of humans in response to endurance exercise training may not be substantially manifested by splitting/intussusception.

Sprouting angiogenesis in skeletal muscle of rats induced by surgical extirpation of a synergistic muscle was associated with an increase of abluminal EC processes, higher rates of EC mitosis (detected by a higher frequency of bromodeoxyuridine 
incorporation), and focal breakage of the pericapillary BM (36). In human muscle biopsies, we only occasionally observed abluminal processes, the capillaries were continuously surrounded by an intact pericapillary BM, and the nucleus density $(\mathrm{Vv})$ in capillary-associated ECs was not different between pre- and postexercise biopsies $(-9 \%)$.

During sprouting angiogenesis induced by extirpation of the tibialis anterior muscle of rats, a higher PC coverage of capillaries $(+42 \%)$ in the extensor digitorum longus muscle (EDL) was observed than of those in the untreated contralateral EDL (14). Accordingly, the PC coverage of capillaries was higher in postexercise VL biopsies than in preexercise biopsies (study 1, +17\%; AR in study 2, +13\%). The findings of the two studies suggest a proangiogenic effect of PCs on capillary ECs in skeletal muscles of rats and humans after an increase in contractility. The interaction of the two cell types might be communicated by the high number of PC projections (i.e., PEGs) inserting into EC pockets (i.e., sockets) in skeletal muscles as shown here and similar to those in rats $(13,31)$.

Given this close spatial relationship between PCs and ECs, it seems reasonable that both cell types communicate intensively. The existence of mechanical contacts in form of gap junctions between ECs and PCs is controversial [reviewed in (2)].

Pericytes have phagocytic activity and are involved in the control or permeability of the blood-brain barrier and for the regulation of blood flow through capillaries (8). It was furthermore speculated that PCs also contribute to the realization of EC-mediated angiogenesis (16). Actually, there was a significant reduction in PC coverage during early phases of angiogenesis in skeletal muscles of rats subjected to chronic electrical stimulation, which suggested an antiangiogenic effect of PCs in this experimental model (13). In contrast, long-term peripheral vasodilation (prazosin model) or prolonged stretch (extirpation model) was accompanied by an increase in PC coverage (14). Thus the role of PCs in controlling physiological angiogenesis obviously depends on the nature of the initial stimulus (14) because sprouting (16) and splitting angiogenesis as well as subsequent capillary remodeling require a dynamic interaction of PCs with ECs. The details of the mutual interaction is widely unknown so far.

Recently, it was shown that the PC pool in the skeletal muscles of mice consists of two distinct populations $(5,6)$. The first PC subtype (type 1), which does not express the intermediate filament nestin, may differentiate into fat cells, whereas the second PC subtype (type 2), which expresses nestin, is part of the stem cell pool for muscle fibers (8) and may regulate angiogenesis (6). However, the contribution of the two PC subtypes to the various angiogenesis modes must be characterized in more detail, especially in human skeletal muscle.

Taken together, an inconsistent picture emerges from the comparison of the structural indicators of skeletal muscle capillaries derived from animal studies and our analysis of human skeletal muscle biopsies derived from study participants undergoing endurance training: some findings are divergent (abluminal EC processes, EC mitosis, breakage of the BM), whereas other changes are aligned (PC coverage). Thus the question of whether exercise-induced angiogenesis in humans is implemented by sprouting cannot be unequivocally answered at this point.
Needle biopsies of human skeletal muscle are too small to facilitate the production of a number of randomized sections sufficient for an isotropic, uniform random sampling strategy (32), which would be required for a formally correct stereological analysis. Nevertheless, the morphometric findings represent acceptable approximations. We furthermore underscore that the values for $\mathrm{T}$ of compartments presented in this investigation represent only quantitative structural descriptors of the capillary ultrastructure and do not provide any evidence of cause/effect relationships between the capillary phenotype and angiogenesis. For a functional interpretation of the findings (e.g., the possible relation of the BM to oxygen and substrate diffusion), other indicators such as the harmonic mean-based thickness have to be ascertained.

In summary, the findings from our study on the ultrastructure of capillaries in human VL biopsies after training indicate that increased PC coverage of capillaries and BM thinning are related to exercise-induced angiogenesis, whereas EC thickening is induced by the training stimulus.

\section{ACKNOWLEDGMENT}

We thank Barbara Krieger for her support with graphical processing of the TEM micrographs.

\section{GRANTS}

Support for this study was provided by Swiss National Science Foundation Grant 320030-144167.

\section{DISCLOSURES}

No conflicts of interest, financial or otherwise, are declared by the authors.

\section{AUTHOR CONTRIBUTIONS}

O.B., H.H.H., and S.A.T. conception and design of research; J.G., A.O., and F.G. performed experiments; O.B., J.G., S.F., E.T., C.M., A.O., and F.G. analyzed data; O.B., S.F., H.H.H., and S.A.T. interpreted results of experiments; O.B. prepared figures; O.B. drafted manuscript; O.B., J.G., S.F., E.T., C.M., A.O., F.G., H.H.H., and S.A.T. edited and revised manuscript; O.B., J.G., S.F., E.T., C.M., A.O., F.G., H.H.H., and S.A.T. approved final version of manuscript;

\section{REFERENCES}

1. Andersen P, Henriksson J. Capillary supply of the quadriceps femoris muscle of man: adaptive response to exercise. J Physiol 270: 677-690, 1977.

2. Armulik A, Genove G, Betsholtz C. Pericytes: developmental, physiological, and pathological perspectives, problems, and promises. Dev Cell 21: 193-215, 2011.

3. Baum O, Da Silva-Azevedo L, Willerding G, Wöckel A, Planitzer G, Gossrau R, Pries AR Zakrzewicz A. Endothelial NOS is main mediator for shear stress-dependent angiogenesis in skeletal muscle after prazosin administration. Am J Physiol Heart Circ Physiol 287: H2300-H2308, 2004.

4. Baum O, Vieregge M, Koch P, Gul S, Hahn S, Huber-Abel FA, Pries AR, Hoppeler H. Phenotype of capillaries in skeletal muscle of nNOSknockout mice. Am J Physiol Regul Integr Comp Physiol 304: R1175R1182, 2013.

5. Birbrair A, Zhang T, Wang ZM, Messi ML, Mintz A, Delbono O. Pericytes: multitasking cells in the regeneration of injured, diseased, and aged skeletal muscle. Front Aging Neurosci 6: 245, 2014.

6. Birbrair A, Zhang T, Wang ZM, Messi ML, Olson JD, Mintz A, Delbono O. Type-2 pericytes participate in normal and tumoral angiogenesis. Am J Physiol Cell Physiol 307: C25-C38, 2014.

7. Bouchard C, Rankinen T. Individual differences in response to regular physical activity. Med Sci Sports Exerc 33: S446-S451; discussion S452S453, 2001.

8. Cappellari O, Cossu G. Pericytes in development and pathology of skeletal muscle. Circ Res 113: 341-347, 2013. 
9. Dawson JM, Hudlicka O. The effects of long term administration of prazosin on the microcirculation in skeletal muscles. Cardiovasc Res 23 . 913-920, 1989.

10. Djonov V, Baum O, Burri PH. Vascular remodeling by intussusceptive angiogenesis. Cell Tissue Res 314: 107-117, 2003.

11. Ebina T, Hoshi N, Kobayashi M, Kawamura K, Nanjo H, Sugita A, Sugiyama T, Masuda H, Xu C. Physiological angiogenesis in electrically stimulated skeletal muscle in rabbits: characterization of capillary sprouting by ultrastructural 3-D reconstruction study. Pathol Int 52: 702-712, 2002.

12. Egginton S, Hudlicka $\mathbf{O}$. Early changes in performance, blood flow and capillary fine structure in rat fast muscles induced by electrical stimulation. J Physiol 515: 265-275, 1999.

13. Egginton S, Hudlicka O, Brown MD, Graciotti L, Granata AL. In vivo pericyte-endothelial cell interaction during angiogenesis in adult cardiac and skeletal muscle. Microvasc Res 51: 213-228, 1996.

14. Egginton S, Zhou AL, Brown MD, Hudlicka O. The role of pericytes in controlling angiogenesis in vivo. Adv Exp Med Biol 476: 81-99, 2000.

15. Egginton S, Zhou AL, Brown MD, Hudlicka O. Unorthodox angiogenesis in skeletal muscle. Cardiovasc Res 49: 634-646, 2001.

16. Gerhardt H, Betsholtz C. Endothelial-pericyte interactions in angiogenesis. Cell Tissue Res 314: 15-23, 2003.

17. Hansen-Smith FM, Hudlicka O, Egginton S. In vivo angiogenesis in adult rat skeletal muscle: early changes in capillary network architecture and ultrastructure. Cell Tissue Res 286: 123-136, 1996.

18. Hoier B, Hellsten Y. Exercise-induced capillary growth in human skeletal muscle and the dynamics of VEGF. Microcirculation 21: 301-314, 2014.

19. Hoppeler H, Howald H, Conley K, Lindstedt SL, Claassen H, Vock P, Weibel ER. Endurance training in humans: aerobic capacity and structure of skeletal muscle. J Appl Physiol 59: 320-327, 1985.

20. Hoppeler H, Luthi P, Claassen H, Weibel ER, Howald H. The ultrastructure of the normal human skeletal muscle. A morphometric analysis on untrained men, women and well-trained orienteers. Pflugers Arch 344: 217-232, 1973.

21. Hudlicka O. Is physiological angiogenesis in skeletal muscle regulated by changes in microcirculation? Microcirculation 5: 7-23, 1998.

22. Ingjer F. Effects of endurance training on muscle fibre ATP-ase activity, capillary supply and mitochondrial content in man. J Physiol 294: 419432, 1979.

23. Ortenblad N, Westerblad H, Nielsen J. Muscle glycogen stores and fatigue. J Physiol 591: 4405-4413, 2013.

24. Perusse L, Rankinen T, Hagberg JM, Loos RJ, Roth SM, Sarzynski MA, Wolfarth B, Bouchard C. Advances in exercise, fitness, and performance genomics in 2012. Med Sci Sports Exerc 45: 824-831, 2013.
25. Phillips BE, Williams JP, Gustafsson T, Bouchard C, Rankinen T, Knudsen S, Smith K, Timmons JA, Atherton PJ. Molecular networks of human muscle adaptation to exercise and age. PLoS Genet 9: e1003389, 2013.

26. Prior SJ, Hagberg JM, Paton CM, Douglass LW, Brown MD, McLenithan JC, Roth SM. DNA sequence variation in the promoter region of the VEGF gene impacts VEGF gene expression and maximal oxygen consumption. Am J Physiol Heart Circ Physiol 290: H1848H1855, 2006.

27. Rivilis I, Milkiewicz M, Boyd P, Goldstein J, Brown MD, Egginton S, Hansen FM, Hudlicka O, Haas TL. Differential involvement of MMP-2 and VEGF during muscle stretch- versus shear stress-induced angiogenesis. Am J Physiol Heart Circ Physiol 283: H1430-H1438, 2002.

28. Rosler K, Hoppeler H, Conley KE, Claassen H, Gehr P, Howald H. Transfer effects in endurance exercise. Adaptations in trained and untrained muscles. Eur J Appl Physiol Occup Physiol 54: 355-362, 1985.

29. Schrauwen-Hinderling VB, Hesselink MK, Schrauwen P, Kooi ME. Intramyocellular lipid content in human skeletal muscle. Obesity (Silver Spring) 14: 357-367, 2006.

30. Suter E, Hoppeler H, Claassen H, Billeter R, Aebi U, Horber F, Jaeger P, Marti B. Ultrastructural modification of human skeletal muscle tissue with 6-month moderate-intensity exercise training. Int J Sports Med 16: 160-166, 1995.

31. Tilton RG, Kilo C, Williamson JR. Pericyte-endothelial relationships in cardiac and skeletal muscle capillaries. Microvasc Res 18: 325-335, 1979.

32. Vock R, Weibel ER, Hoppeler H, Ordway G, Weber JM, Taylor CR. Design of the oxygen and substrate pathways. V. Structural basis of vascular substrate supply to muscle cells. J Exp Biol 199: 1675-1688, 1996.

33. Weibel E. Stereological methods. Practical methods for biological morphometry. London: Academic Press, 1979.

34. Williamson JR, Hoffmann PL, Kohrt WM, Spina RJ, Coggan AR, Holloszy O. Endurance exercise training decreases capillary basement membrane width in older nondiabetic and diabetic adults. J Appl Physiol 80: 747-753, 1996.

35. Zhou A, Egginton S, Hudlicka O, Brown MD. Internal division of capillaries in rat skeletal muscle in response to chronic vasodilator treatment with alpha1-antagonist prazosin. Cell Tissue Res 293: 293-303, 1998.

36. Zhou AL, Egginton S, Brown MD, Hudlicka O. Capillary growth in overloaded, hypertrophic adult rat skeletal muscle: an ultrastructural study. Anat Rec 252: 49-63, 1998. 